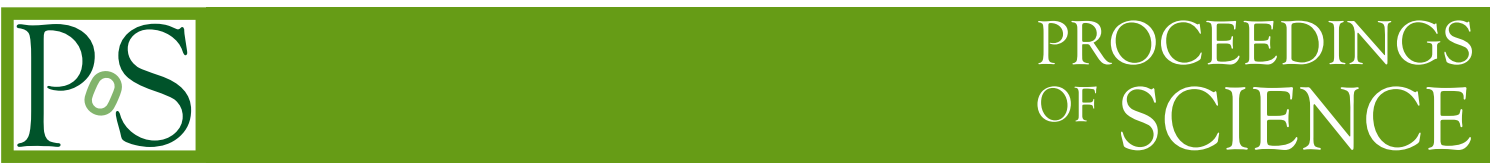

\title{
Gamma-Ray Burst observations with Fermi
}

\section{E. Bissaldi*(1) , F. Longo $^{(2,3)}$, N. Omodei ${ }^{(4)}$, G. Vianello ${ }^{(4)}$, A. von Kienlin ${ }^{(5)}$ on behalf of the Fermi/LAT Collaboration}

(1) Istituto Nazionale di Fisica Nucleare, Sezione di Bari, Bari, Italy

(2) Dipartimento di Fisica, Università degli Studi di Trieste, Trieste, Italy

(3) Istituto Nazionale di Fisica Nucleare, Sezione di Trieste-Udine, Trieste, Italy

(4) Stanford University, Stanford, CA, United States

(5) Max-Planck-Institut für extraterrestrische Physik, Garching, Germany

E-mail: Elisabetta.Bissaldi@ba.infn.it

After seven years of science operation, the Fermi mission has brought great advances in the study of Gamma-ray Bursts (GRBs). Over 1600 GRBs have been detected by the Gamma-ray Burst Monitor, and more than 100 of these are also detected by the Large Area Telescope above 30 $\mathrm{MeV}$.

We will give an overview of these observations, presenting the common properties in the GRB temporal and spectral behavior at high energies. We will also highlight the unique characteristics of some individual bursts. The main physical implications of these results will be discussed, along with open questions regarding GRB modeling in their prompt and temporally-extended emission phases.

The 34th International Cosmic Ray Conference,

30 July- 6 August, 2015

The Hague, The Netherlands

\footnotetext{
*Speaker.
} 


\section{The Fermi mission}

The contribution of the Fermi mission to the study of the high-energy emission from GRBs has been of great importance over the past seven years. Thanks to its $12 \mathrm{NaI}$ detectors and two BGO detectors, sensitive in the $8 \mathrm{keV}-1 \mathrm{MeV}$ and $250 \mathrm{keV}-40 \mathrm{MeV}$ energy ranges, respectively, and to an almost $4 \pi$ sr Field of View (FoV), the Gamma-Ray Burst Monitor (GBM) [1] has detected over 1600 bursts at the time of this writing.

A great number of GBM GRBs has also been detected at higher energies by the Large Area Telescope (LAT) [2]. This pair production gamma-ray telescope is sensitive from $\sim 30 \mathrm{MeV}$ to $>300 \mathrm{GeV}$ and features a FoV of $2.4 \mathrm{sr}$ at $1 \mathrm{GeV}$, a low dead time per event ( $27 \mu \mathrm{s})$ and the largest effective area for gamma-ray space satellites at $\mathrm{GeV}$ energies. The actual LAT performances can be found in [3]. This allows the LAT to get a larger number of GRB detections at energies $>\sim 30$ $\mathrm{MeV}$ ( $\sim 9$ GRBs/year) with respect to its predecessor EGRET (5 GRBs in 10 years) or to AGILE (7 GRBs in 8 years), and in rough agreement with the pre-launch expectations [4].

\section{The Fermi/GBM GRB catalogs}

The most important observables of the GBM detected GRBs are summarized in a series of catalogs. The latest catalog issue, the second GRB catalog [5], lists 953 GRBs detected in the first four years of the mission, and provides their locations, the main characteristics of the prompt emission, the durations, peak fluxes and fluences. A sky distribution of these GRBs is shown in Figure 1. An accompanying second spectral catalog [6] provides information on the systematic spectral analysis of nearly all GRBs listed in the trigger catalog. Time-integrated fluence and peak flux spectra are presented for all GRBs. Moreover, a separate catalog reporting time resolved spectral analysis of the brightest GBM GRBs is in preparation. Preliminary results of this type of analysis on eight bright GRBs can be found in [7]. All of these catalogs are an official product of

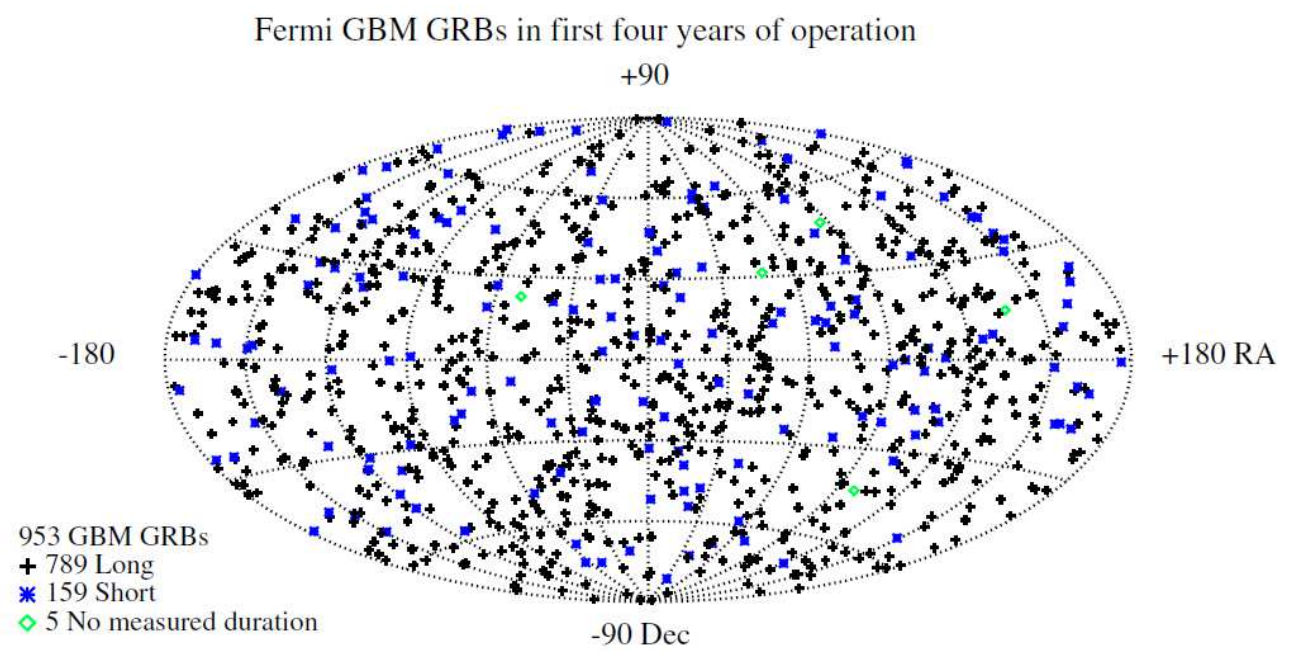

Figure 1: Sky distribution of GBM triggered GRBs in celestial coordinates. Crosses indicate long GRBs (T90 > 2 s); asterisks indicate short GRBs. Taken from [5]. 
the Fermi/GBM science team, and the data files containing the complete results are available from the High-Energy Astrophysics Science Archive Research Center (HEASARC) [8].

The GRB sample analysis presented in the second catalog establishes the conclusions of the first catalog, which covered two years of data acquisition [9]. The rate of burst detections $(\sim 240$ GRBs/yr), which is only slightly smaller compared to the rate of the BATSE instrument $(\sim 300$ GRBs/yr), can be explained by the GBM additional range of trigger timescales (primarily the $2 \mathrm{~s}$ and $4 \mathrm{~s}$ timescales), which are compensating for the higher burst detection threshold of GBM $(\sim 0.7$ versus $\sim 0.2$ photons $\mathrm{cm}^{-2} \mathrm{~s}^{-1}$ for BATSE). The distribution of GBM durations is consistent with the well-known bimodality measured previously and the fraction of about $17 \%$ of short GRBs in the GBM sample is somewhat smaller than detected by BATSE, which is mainly attributed to the GBM capability to trigger on longer timescales.

The spectral properties of the bursts presented in the spectral catalog are described both from a time-integrated and a peak-flux analysis. Four photon model fits are applied to each selection, resulting in more than 7500 spectral fits. Model comparison techniques were applied to select the most statistically preferred model for each GRB and the resulting GRB parameter distributions were investigated extensively. Small discrepancies between the first GBM spectral catalog [10] and the second spectral catalog are mainly due to enhanced and modified criteria in determining the GRB samples, updated analysis software and response matrices and the usage of different statistical criteria.

\section{The Fermi/LAT GRB catalog}

The first Fermi/LAT GRB catalog [11] covers three years of observations, from August 2008 to July 2011. In this period GBM detected $\sim 750$ GRBs, with around half of them in the LAT FoV. The two detection algorithms used by LAT are: (1) a standard likelihood algorithm, providing both detection and localization with $<1$ deg accuracy, using the post-launch reconstruction method "Pass 6 v3 Transient" [12] for events above $100 \mathrm{MeV}$; and (2) a counting analysis using the LAT Low-Energy data (LLE) [13]. The LLE technique features a large effective area starting at $~ 30$ $\mathrm{MeV}$ but no localization capability. While algorithm (1) detected and localized $28 \mathrm{GRBs}$, algorithm (2) uncovered 7 more bursts, for a total of 35 GRBs.

While the number of GRBs detected by LAT is a small fraction of the total number of GBM GRBs in the LAT FoV, this sample allows us to uncover unique features of GRBs emerging only at high energies.

\subsection{Energetics}

Since LAT observations are photon-limited rather than background-limited, the detection efficiency is directly related to the counts fluence of the source. This is an important difference with respect to GBM, which is background limited and for which the peak flux of the source is more relevant. Of course, the low-energy fluence is highly correlated with the high-energy fluence. The fact that LAT detects preferentially GRBs with a high low-energy fluence is therefore not surprising. The typical ratio between the high-energy fluence (above $100 \mathrm{MeV}$ ) and the low-energy fluence $(10 \mathrm{keV}-1 \mathrm{MeV})$ is $\sim 0.1$. 
It is interesting to note that the catalog presents four hyper-energetic bursts for which the ratio greatly exceeds the typical value, being closer or even above 1 . The same conclusion can be reached taking the ratio of the rest frame total energy $E_{i s o}$ in the two energy bands, which demonstrates that this is not an effect of the distance of these bursts, which are distributed between redshift 0.9 and 4.35 .

\subsection{Delayed and temporally extended emission}

The emission above $100 \mathrm{MeV}$ is systematically delayed with respect to the low-energy emission. When using $T_{05}$ as a measure of the onset of the emission for both the 10-300 keV energy range (from [9]) and the $100 \mathrm{MeV}-10 \mathrm{GeV}$ energy range, it is clear that the latter is systematically larger than the former. Also, the duration of the high-energy emission appears to be systematically longer, and features a smooth decaying phase after the end of the low-energy prompt emission.

Such decaying phase is well described by a power law with typical decay index $\alpha_{L} \simeq-1$, pointing to a different physical origin with respect to the spiky prompt emission. This value is foreseen by the standard afterglow model for an adiabatic expansion of the fireball. A radiative expansion would foresee a decay with an index of 10/7, which is not observed in the LAT data.

In a few cases, a broken power law gives a better description of the temporally extended data, and the time of the temporal decay break is usually found after the end of the low-energy emission, as measured by $\mathrm{T}_{90}$.

\subsection{High-energy photons}

The LAT has observed photons up to $95 \mathrm{GeV}$ coming from bright GRBs, which in the case of high-redshift GRBs can become more than $100 \mathrm{GeV}$ in the rest frame of the progenitor of the burst. This result poses a big challenge for the efficiency of the particle acceleration mechanisms, especially when considering the fact that some of these high-energy events have been detected within seconds since the start of the low-energy emission. In the context of the standard fireball model [14], the presence of such high-energy photons constrains also the bulk Lorentz factor of the emitting shells to be $\Gamma>1000$ in some cases, a value much higher than what previously thought.

High-energy photons from high-redshift GRBs also allow to constrain the opacity of the Universe connected with the interaction of the $>10 \mathrm{GeV} \gamma$-rays with optical and UV photons of the Extragalactic Background Light (EBL). In the case of the short GRB 090510, the short time delay observed between low and high-energy events can be used to place tight limits on the energy dependence of the speed of light, which is postulated for example by some quantum gravity theories [15].

\subsection{Broad-band spectroscopy}

Fermi is an exceptional observatory for GRB spectroscopy of the prompt emission. By combining GBM and LAT data, we can achieve an unprecedented spectral coverage spanning almost 7 decades in energy, from $10 \mathrm{keV}$ up to $300 \mathrm{GeV}$. This feature was exploited in the catalog by performing a broad-band spectral analysis of all the GRBs contained in the sample. Before Fermi most of the GRB spectra were well described by the empirical Band model [16], which has become the de-facto "standard GRB model". The spectra of all the brightest bursts inside the LAT FoV 
present, on the contrary, significant deviations from a Band function, requiring additional components such as power laws, high-energy cutoffs, or both. Other GRBs, observed at low off-axis angles, and with a corresponding high effective area, show deviations as well. We conclude that the empirical Band model seems to be not sufficient to describe all the spectral features of LAT GRBs. Unfortunately, there is no common recipe, and different components can be required depending on the particular event. This calls for a better broad-band modeling of the prompt spectra of GRBs, opening new questions and prompting new theoretical developments.

\subsection{The afterglow of LAT-detected GRBs}

A sub-sample of LAT-detected GRBs have been studied at other wavelengths, in particular during their afterglow emission. A systematic study published by [17] shows that in many ways the properties of the afterglow of LAT bursts are typical of the general afterglow population, but the ratio between the luminosity of the prompt emission and the luminosity of the afterglow is larger. Therefore, either their prompt emission is more efficient in producing gamma-rays, or, conversely, their afterglows are somehow suppressed. In two cases, GRB 090510 and GRB 110731A, Swift and other instruments observed the afterglow when the high-energy extended emission was still detectable by the LAT. Dedicated broadband studies, from optical wavelengths to gamma-rays, showed that the emission is compatible with being from external shocks [18, 19]. In another case, GRB 100728A, high-energy emission was detected by the LAT only in correspondence with an $\mathrm{X}$-ray flare, which was successfully modeled from $\mathrm{X}$ to gamma-ray energies as internal shock emission [20].

\section{The extremely bright GRB 130427A}

The observations of the exceptionally bright GRB 130427A by Fermi provide further constraints on the GRB phenomenon and their emission processes. It is one of the most energetic GRBs ever observed. The initial pulse up to 2.5 seconds is possibly the brightest well-isolated pulse observed to date. A fine time resolution spectral analysis led by the Fermi/GBM Team [21] highlighted how difficult it is for any of the existing models to account for all of the observed spectral and temporal behaviors simultaneously.

Furthermore, GRB 130427A had the largest fluence, highest observed energy photon (95 $\mathrm{GeV}$ ), longest gamma-ray duration (20 hours), and one of the largest isotropic energy releases ever observed from a GRB. The temporal and spectral analysis of GRB 130427A presented by the Fermi/LAT Team in [22] challenge the widely accepted model that the non-thermal high-energy emission in the afterglow phase of GRBs is synchrotron emission radiated by electrons accelerated at an external shock.

\section{Towards the second LAT GRB catalog}

All LAT GRB detections are constantly maintained and kept up-to-date on two publicly available GRB tables $[23,24]$. They list 100 GRBs at the time of this writing. The Fermi/LAT collaboration is actively working to produce the second version of its GRB catalog, probably covering six years into the mission. This catalog will contain more GRBs, not only due to an extended period 
of data acquisition, but also due to (1) renewed algorithms to search on a wider angular region centered on GBM GRB trigger positions and to (2) new LAT data selection, with larger effective area both at low and at high energies.

\subsection{New GRB detection algorithm}

Starting from the results obtained in the first LAT GRB catalog and thanks to recent developments in the understanding of the systematic errors on GBM localizations [25], we are currently developing and testing a new detection algorithm which increases the number of detections by $>45 \%$ and was first presented in [26]. It consists of 10 searches running in parallel over time intervals logarithmically spaced from the trigger time to $10 \mathrm{ks}$ after that. In case of a GBM trigger, for each of these time intervals the algorithm creates a grid in equatorial coordinates and with a spacing of $0.7 \mathrm{deg}$, covering a finding map of $30 \times 30 \mathrm{deg}$, thus covering the GBM position uncertainty. For each point of the grid, a likelihood analysis is performed and a test statistics (TS) value is computed Finally, the maximum of the TS in the map is considered as the best guess for the position of the new transient, and marked for further position optimization. A new likelihood analysis is performed on the best position found. If the TS from this final analysis is above a certain threshold, we consider it a new detection (a $5 \sigma$ detection corresponds to TS $\sim 28$ ).

\subsection{GRBs with Pass 8}

Since 2010, the Fermi/LAT collaboration is developing a comprehensive revision of the eventlevel analysis, known as "Pass 8". It provides a full reprocessing of the entire mission data-set,

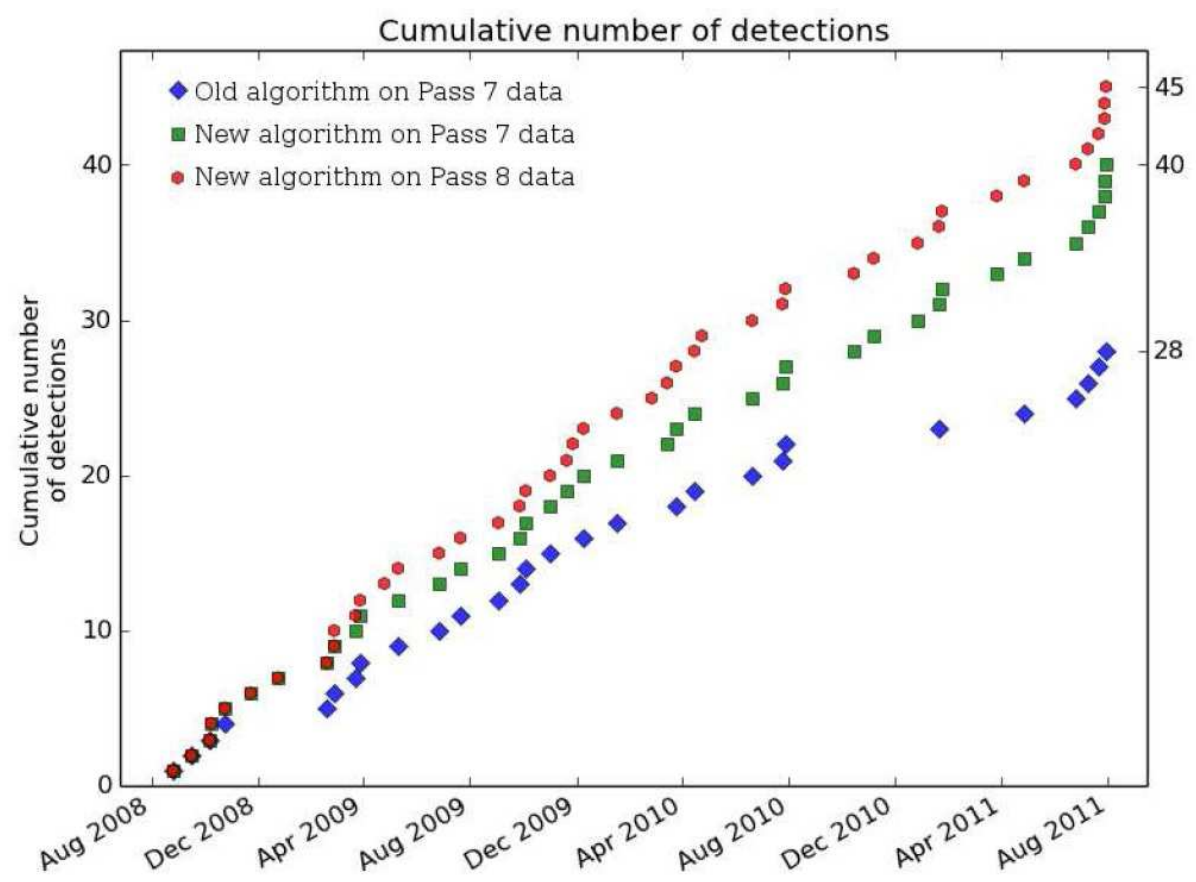

Figure 2: Cumulative detections for the time span covered by the LAT GRB catalog. The new analysis yields $45 \%$ more detections than the one used in the catalog when run on the same data (blue and green), and $60 \%$ more with Pass 8 data (red). Taken from [26]. 
including improved event reconstruction, a wider energy range, better energy measurements, and significantly increased effective area. In addition, the events have been evaluated for their measurement quality in both position and energy. As of the release date (June 24, 2015), the Fermi Sciene Support Center (FSSC) is now serving Pass 8 LAT data for analysis [27].

Using its preliminary achievements, the LAT collaboration re-analyzed the prompt phase of ten bright GRBs previously detected by the LAT [28], finding four new gamma rays with energies greater than $10 \mathrm{GeV}$ in addition to the seven previously known. Among these four there is a 27.4 GeV gamma-ray from GRB 080916C, which, at a redshift of 4.35, makes it the gamma-ray with the highest intrinsic energy $(147 \mathrm{GeV})$ detected so far from a GRB.

If we combine the specialized search algorithm with the Pass 8 event selection, we largely enhance the detection of faint high-energy GRB counterparts, increasing the efficiency of detection by more than $60 \%$, yielding more than 100 bursts over the time span of the Fermi mission (see Figure 2). The analysis and characterization of this new sample is currently in progress. When completed, it will hopefully help to settle some open questions which we could not firmly establish in the first catalog due to the limited statistics, such as the existence of a separate population of hyper-energetic events, the nature of the fireball expansion (adiabatic vs. radiative), or how common are spectral cutoffs.

\section{Acknowledgments}

The Fermi-LAT Collaboration acknowledges support for LAT development, operation and data analysis from NASA and DOE (United States), CEA/Irfu and IN2P3/CNRS (France), ASI and INFN (Italy), MEXT, KEK, and JAXA (Japan), and the K.A. Wallenberg Foundation, the Swedish Research Council and the National Space Board (Sweden). Science analysis support in the operations phase from INAF (Italy) and CNES (France) is also gratefully acknowledged.

\section{References}

[1] C. Meegan et al., ApJ 702, 791 (2009)

[2] W. B. Atwood et al., ApJ 697, 1071 (2009)

[3] http://www.slac.stanford.edu/exp/glast/groups/canda/lat_Performance.htm

[4] D. L. Band et al., ApJ 701, 1673 (2009)

[5] A. von Kienlin et al., ApJS 211, 13 (2014)

[6] D. Gruber et al., ApJS 211, 12 (2014)

[7] H. F. Yu et al., $A \& A$ 573, 81 (2015)

[8] http://fermi.gsfc.nasa.gov/ssc/data/access/gbm/

[9] W. S. Paciesas et al., ApJS 199, 18 (2012)

[10] A. Goldstein et al., ApJS 199, 19 (2012)

[11] M. Ackermann et al., ApJS 209, 11 (2013)

[12] R. Rando for the Fermi/LAT Collaboration, arXiv e-print 0907.0626 (2009)

[13] V. Pelassa et al., e-print arXiv: 1002.2617 (2010) 
[14] T. Piran, Physics Reports 314, 575 (1999)

[15] V. Vasileiou et al., PRD 87, 12 (2013)

[16] D. L. Band et al., ApJ 413, 281 (1993)

[17] J. L. Racusin et al., ApJ 738, 138 (2011)

[18] M. De Pasquale et al., ApJL 709, L146 (2010)

[19] M. Ackermann et al., ApJ 763, 71 (2013)

[20] A. A. Abdo et al., ApJL 734, L27 (2011)

[21] R. Preece et al., Science 343, 51 (2014)

[22] M. Ackermann et al., Science 343, 42 (2014)

[23] http://fermi.gsfc.nasa.gov/ssc/observations/types/grbs/lat_grbs/table.php

[24] http://www.asdc.asi.it/grblat/

[25] V. Connaughton et al., ApJS 216, 32 (2015)

[26] G. Vianello et al., e-print arXiv: $\mathbf{1 5 0 2 . 0 3 1 2 2}$ (2015)

[27] http://fermi.gsfc.nasa.gov/ssc/data/analysis/documentation/Pass8_usage.html

[28] W. B. Atwood et al., ApJ 774, 76 (2013) 